\title{
CIENCIAS BIOLÓGICAS Y LÍNEA PROFESIONAL: OPINIÓN DE ENFERMERAS DOCENTES PARA LA FORMACIÓN DE COMPETENCIAS PROFESIONALES ${ }^{1}$
}

\section{BIOSCIENCES AND DISCIPLINE-RELATED SUBJECTS: VIEWS OF NURSE TRAINING TEACHERS ON PROFESSIONAL COMPETENCES}

\author{
Mónica Illesca Pretty* \\ Ximena Osorio Spuler ${ }^{* *}$ \\ JEANETTE JARA BADILLA** \\ Mirtha Cabezas GonZÁlez ${ }^{* * *}$
}

\begin{abstract}
RESUMEN
Objetivo: Conocer la opinión de las Enfermeras docentes respecto a los factores asociados a la integración de Ciencias Básicas con la línea Profesional en el plan de estudio de la carrera de Enfermería. Método: Se realizó en el 2011 una investigación cualitativa a través de un estudio intrínseco de casos. Los informantes claves fueron 16 docentes de la Carrera de Enfermería que realizaban actividades de supervisión clínica, mínimo tres años de ejercicio docente, y que otorgaron Consentimiento Informado. La recolección de datos se hizo en grupos focales de 8 integrantes cada uno. El análisis siguió un esquema de reducción progresiva: disposición, transformación y obtención de conclusiones verificables, validándose por triangulación de investigadores. Para esos efectos se utilizó el programa ATLAS.ti. Resultados: Se encontraron 111 unidades de significado, originando tres categorías: "Propuestas para la integración", "Factores que dificultan la integración" y "Relación Ciencias Básicas con la profesión". Al final del proceso surge un dominio cualitativo "Consideraciones para favorecer la integración entre Bases Morfológicas I y II con la Línea Profesional en el desarrollo de competencias disciplinares para el futuro desempeño laboral”. Conclusión: No se verificó la integración de Ciencias Básicas con la línea Profesional. Los informantes identifican sólo factores que dificultan la integración.
\end{abstract}

Palabras clave: Educación en enfermería, competencia profesional, disciplinas de las ciencias biológicas.

\begin{abstract}
Objective: To know the opinion of teaching nurses regarding factors associated with integrating basic science to the discipline-ralated subjects present in the nursing major curriculum. Method: A qualitative study was conducted in 2011 through intrinsic case study. The key informants were 16 teachers of Nursing School who were involved in active clinical supervision, had at least three years of teaching practice and had signed an informed consent. Data collection was done through focus groups of 8 members each. The analysis followed a progres-

\footnotetext{
${ }^{1}$ Proyecto de Investigación DI10-0036. Universidad de La Frontera.

* Enfermera. Doctora en Salud. Departamento Medicina Interna. Facultad de Medicina. Universidad de La Frontera. Manuel Montt 112 Temuco, Chile. E-mail: monica.illesca@ufrontera.cl

${ }^{* *}$ Enfermera. Mg. Enfermería. Departamento Enfermería. Facultad de Medicina. Universidad de La Frontera. Manuel Montt 112 Temuco, Chile. E-mail: ximena.osorio@ufrontera.cl

${ }^{* * *}$ Enfermera. Licenciada en Enfermería. Departamento Enfermería. Facultad de Medicina. Universidad de La Frontera. Manuel Montt 112 Temuco, Chile.E-mail: jeanette.jara@ufrontera.cl

**** Químico-Farmacéutico. Departamento Ciencias Preclínicas. Facultad de Medicina. Universidad de La Frontera. Manuel Montt 112 Temuco, Chile.E-mail:mirtha.cabezas@ufrontera.cl
} 
sive reduction scheme: arrangement, processing and obtaining verifiable conclusions, validating triangulation of researchers. The ATLAS.ti program was used for this purpose. Results: 111 units of meaning were found, resulting in three categories: "Proposals for integration", "Factors that make integration difficult" and "Basic Sciences relationship with the nursing profession". At the end of the process a qualitative domain emerged: "Considerations to promote integration between Morphological Bases I and II with the professional line in the development of disciplinary competences for future work performance". Conclusion: The integration of Basic Science to the Professional line was not verfied. Informants identified factors that hinder integration only.

Key words: Nursing education, professional competence, biological science disciplines.

Fecha recepción: 30/03/15 Fecha aceptación: 15/07/16

\section{INTRODUCCIÓN}

La forma de cómo se obtienen los conocimientos es primordial para que éstos se constituyan en un aprendizaje significativo, es decir entender y comprender el significado, lo que queda demostrado cuando el sujeto puede ponerlo en práctica para resolver problemas (1).

Específicamente, en la función asistencial, las/os enfermeras/os deben incorporar en su estructura cognitiva los conocimientos que han aprendido, reflejando las distintas disciplinas básicas de manera integrada. Este proceso presupone la existencia de partes que deben estar relacionadas para constituir un todo, completando un proceso mental, mediante el cual se unen diferentes conocimientos que forman una nueva unidad de síntesis interdisciplinaria con mayor grado de generalización (2).

Lo anterior, referido a la integración, corresponde a una propiedad inherente de todo sistema, que implica unir elementos separados en un conjunto coherente (3), cuyos contenidos suelen tener carácter horizontal o vertical según los nexos que entre ellos se aborden de forma simultánea o consecutivamente a lo largo del Plan de Estudio (4), como son los correspondientes a las denominadas Ciencias Básicas y Ciencias Clínicas (5). Por otro lado, se puede considerar como una vía para solucionar los problemas causados por la explosión de los conocimientos, como consecuencia del impetuoso desarrollo de la ciencia y la técnica $(6,7)$.

Entre los saberes básicos de las/os enfermeras/os están las disciplinas del área biológica, que son unos de los pilares para conocer cómo está constituido el organismo humano, tanto en los aspectos estructurales como funcionales, con el fin último de gestionar los cuidados acorde a los problemas de salud de los individuos (8).

Desde el año 2003 la Carrera de Enfermería de una universidad de la Región de la Araucanía imparte el Plan de Estudio con una filosofía educacional centrada en el estudiante, en sus intereses, necesidades, inquietudes y todo aquello que pueda conducir al alcance de su autorrealización. Además, apoyado en principios de educación del adulto y aprendizaje basado en problemas prioritarios de salud, a través de módulos integrados (9). Ello, al amparo de las concepciones constructivistas, que actualmente se utilizan en educación, para que los saberes adquiridos tengan significado, donde los conocimientos que los discentes ya poseen sean, precisamente, los que les permiten consolidarlos, surgiendo una retroalimentación, nacida desde su propio hacer, con una mirada de mayor complejidad y comprensión (10). Lo anterior significó asumir un nuevo rol en el quehacer de la práctica educativa en diversas dimensiones y niveles en el proceso de cambio (11), desde lo más simple y superficial (a corto plazo) hasta lo más complejo y profundo (a largo plazo), lo que afecta, creencias, valores 
e ideología que modelan las concepciones y prácticas pedagógicas (12).

El Plan de Estudio se estructura a través de cuatro líneas curriculares, correspondiendo la Morfofunción y la Profesional a dos de ellas. La primera incluye las Ciencias Básicas y Preclínicas, entrega las bases de la formación científica que permite entender al ser humano como una persona anátomo-biofuncional. Dentro de las Ciencias Básicas, impartida en los Módulos Integrados Bases
Morfológicas I y II (primer y segundo nivel de la Carrera), se aúnan las asignaturas de Anatomía, Histología y Embriología. La segunda proporciona los fundamentos, habilidades y destrezas necesarias para el desempeño de competencias disciplinares y genéricas e incluye los temas propios de Enfermería con contenidos de Ciencias Básicas, Preclínicas y Sociales (9). Lo anterior se representa en el siguiente esquema:

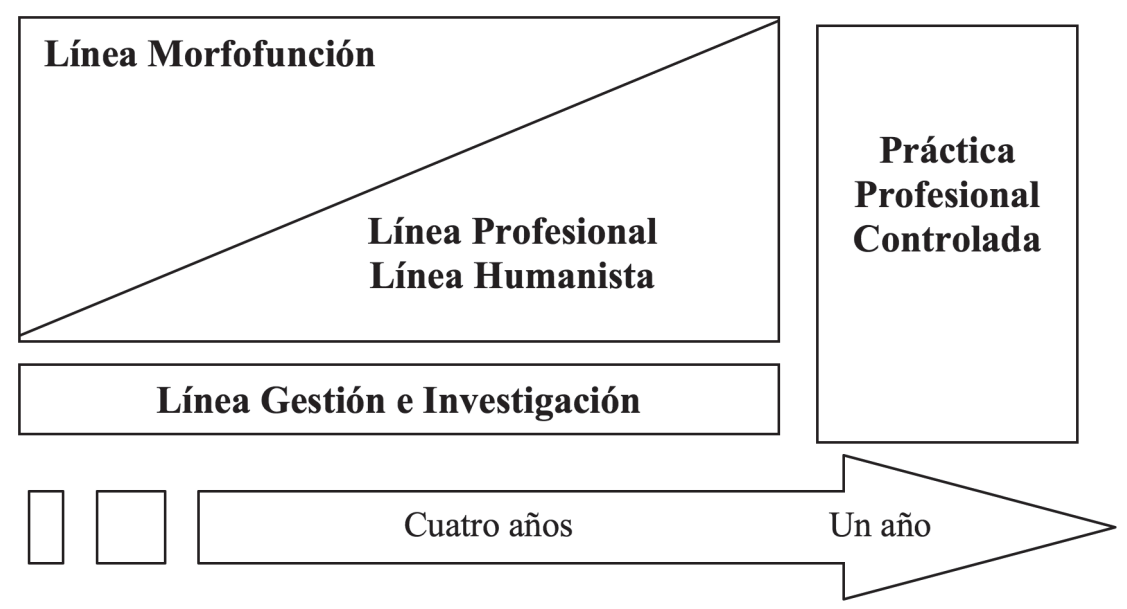

Figura 1. Esquema que representa las líneas curriculares del plan de estudio de la carrera de Enfermería.

En la propuesta pedagógica inicial se planificaron estos módulos de carácter teóricopráctico, centrándose en el análisis de situaciones de aprendizaje de problemas prioritarios de salud, como ya se dijo, con un enfoque holístico, incorporando en su diseño los aspectos biológicos, psicológicos y sociales, los que consideran distintos recursos de aprendizaje como son clases expositivas, tutorías, talleres, laboratorios, demostración y devolución de procedimientos, pasos prácticos, experiencias clínicas, trabajo en terreno, entre otros, con el apoyo de biblioteca, multimedia, utilización de tecnologías de información y comunicación. Asimismo, se sumaron como recurso para consultas específicas, académicos de la Facultad, docentes de otras carreras del área de la salud y profesionales asistenciales. El sistema de evaluación se formuló acorde a la metodología empleada, utilizando técnicas e instrumentos para evaluar proceso y resultado, tanto en los aspectos teórico como práctico (9). Es así como los programas de los Módulos Integrados Bases Morfológicas I y II representan lo estipulado por la Carrera $(13,14)$.

En la Evaluación Institucional, los Módulos Bases Morfológicas I y II, en general están bien conceptuados en todas las dimensiones que considera este proceso: organización, actualización, motivación, aprendizaje logrado, relación interpersonal, evaluaciones y formación integral (15), alcanzando promedios similares al de las demás actividades 
curriculares. Al comparar estos Módulos con las asignaturas equivalentes del antiguo Plan de Estudio se observa que el índice de reprobación ha disminuido de un $8,6 \%$ a un $7,5 \%$ en el Plan actual (16).

Sin embargo, lo satisfactorio de estos datos no concuerda con la realidad observada cuando los estudiantes se enfrentan a la práctica, donde se esperaría que integren y apliquen los contenidos en las actividades curriculares profesionales. Ello ha sido evidenciado por enfermeras docentes, que supervisan las prácticas clínicas, quienes expresan que los estudiantes presentan déficit de conocimientos en forma específica en Anatomía, lo cual debe ser nuevamente revisado de acuerdo a las competencias de la disciplina. Con respecto a Embriología e Histología, manifiestan no observar la aplicabilidad ni la integración entre ellas, como tampoco con la profesión. Lo planteado coincide con las conclusiones de una investigación donde se postula la inexistencia de vinculación entre contenidos de Morfología y los de otras asignaturas (17). Lo anterior provoca dificultades en la didáctica educativa tanto para docentes como estudiantes. En el aspecto teórico, los académicos se ven en la necesidad de repetir tópicos, restando tiempo a los conocimientos que deberían adquirir. En las experiencias clínicas no observan el sustento integrado de la Anatomía, Histología y Embriología en el logro de las competencias disciplinares, significando para los alumnos una sobrecarga adicional, ya que deben estudiar nuevamente los contenidos y hacer la relación con el área profesional.

Existe consenso que la motivación del estudiante durante su formación académica es la aplicación práctica del objeto de estudio; es así que la Anatomía debe ser utilizada de forma inmediata en el quehacer de Enfermería cuando realiza la etapa de valoración en el examen físico (18). Sin los contenidos anatómicos se hace imposible aplicar el Proceso de Enfermería, pues éste requiere de métodos semiológicos, la inspección, palpación, per- cusión y auscultación, como tampoco realizar procedimientos propios de la disciplina al carecer de un marco teórico sobre el cual se fundamenten las acciones $(19,20)$.

En la literatura se observa preocupación por relacionar asignaturas de Morfofunción con las competencias profesionales, existiendo propuestas de modelos educativos para lograr esta necesaria vinculación $(21,22,23)$.

En resumen, se ha identificado el problema a investigar, más aún cuando, a doce años de la puesta en marcha del Plan de Estudio, no existe una evaluación curricular por parte de los académicos, sólo la Institucional, la cual considera criterios generales sin especificidad en relación a las características del modelo pedagógico.

Con el propósito de contribuir a mejorar la calidad en la formación de Enfermeras (os) en lo que se refiere a la integración de las Bases Morfológicas con la Línea Profesional, se formulan los siguientes objetivos: a) develar factores que intervienen en esta integración y b) indagar las competencias específicas de la profesión que se logran con los contenidos de los Módulos Bases Morfológicas I y II.

La trascendencia de esta investigación está dada en que a futuro la Carrera de Enfermería, al disponer de datos actuales y específicos, le permitirá tomar decisiones razonadas en relación a la planificación educativa de los Módulos Integrados, de tal forma de consolidar las competencias disciplinares y genéricas. A su vez, generar estrategias para dar respuesta al medio externo, asegurando de esta forma la calidad en el proceso educativo, asumiendo el compromiso con la sociedad de dotar al país con profesionales necesarios acorde a la realidad emergente. En este sentido, es pertinente citar a quien plantea que el conocimiento y la actitud del profesorado hacia las tareas que constituyen la docencia universitaria, representa uno de los puntos claves de todo el proceso de mejora docente, ya que contribuye a certificar el desempeño del futuro profesional (24). 


\section{MATERIAL Y MÉTODO}

Se optó por el paradigma cualitativo, descriptivo e interpretativo, a través de un estudio intrínseco de casos $(25,26)$, que permitirá a futuro generar cambios en la situación del problema detectado.

El objeto de estudio fueron docentes de la Carrera de Enfermería (2011), conformando una muestra no probabilística, intencionada de casos por criterio y por conveniencia (27) de 16 informantes claves, quienes a partir de sus historias personales individuales y colectivas proporcionaron información válida con relación a los factores que intervienen en la integración de contenidos de Bases Morfológicas con la Línea Profesional, cuyo criterio de inclusión fueron: ser Enfermera, realizar actividades de supervisión clínica, tener como mínimo tres años de ejercicio docente, disponibilidad horaria para asistir a las sesiones de grupos focales y haber firmado el Consentimiento Informado.

Para la recolección de datos se utilizó la técnica cualitativa de grupos focales con una pauta de entrevista semiestructurada (2 grupos de 8 integrantes cada uno), la de análisis de contenido (revisión bibliografía especializada) y la documental (análisis de registros), empleándose notas de campo y grabaciones de voz, con la correspondiente autorización de las entrevistadas. Se obtuvieron desde distintos puntos de vista, realizándose múltiples similitudes de un fenómeno único, en varios momentos, desde diversas perspectivas $y$ procedimientos.

El análisis de datos siguió el esquema de comparaciones constantes (28), no agrupándose en categorías predeterminadas, sino más bien emergiendo de un proceso de razonamiento inductivo. En forma progresiva se realizó la reducción de la información concordante con el objeto de estudio a través de tres fases que conformaron diferentes tipos de operaciones, segmentación y codificación de unidades de significado, identificación de los temas principales o núcleos temáticos emergentes e integración e interpretación de los resultados en vectores cualitativos (29, 30).

- Nivel 1: Identificación de unidades de significado (narraciones textuales de los sujetos participantes) y segmentación de las mismas para su agrupación en categorías descriptivas.

- Nivel 2: A partir de las categorías descriptivas se construyó un sistema de núcleos temáticos emergentes o metacategorías.

- Nivel 3: Como consecuencia del nivel anterior, se identificaron los dominios cualitativos a través de un análisis secuencial y transversal de las metacategorías.

Para estos efectos se utilizó el programa computacional ATLAS.ti. Cabe señalar que bajo este paradigma la recolección de datos y el análisis de ellos fue concurrente (31); la categorización y segmentación fueron dos operaciones que se realizaron simultáneamente porque el criterio usado fue la pertenencia a un determinado concepto o tópico, donde las unidades que hacían referencia a determinada idea se incluyeron en las categorías que se correspondían con esa idea.

Como una forma de asegurar que los resultados de la investigación no sean el producto de una sola fuente o sesgo del investigador, y transformar el dato a una información de la realidad y dar credibilidad al estudio, la rigurosidad científica estuvo determinada por cuatro criterios de rigor: valor de verdad (credibilidad), aplicabilidad (transferibilidad), consistencia (dependencia) y neutralidad (confirmabilidad) (32).

Las consideraciones éticas se resguardaron al ser un Proyecto autorizado por la Dirección de Investigación y Postgrado de la institución a través del Comité para estos efectos. Se trabajó con los principios éticos en investigación (33). 


\section{RESULTADOS}

El análisis de datos de los dos grupos focales siguió un esquema que en forma progresiva generó una reducción de la información concordante con el objeto de estudio, a través de tres niveles:

Nivel 1: Se encontraron 111 unidades de significado relevantes para el estudio agrupados en 3 categorías emergentes: "Propuestas para la integración" (PIN), "Factores que dificultan la integración" (FDI) y "Relación Ciencias Básicas con la profesión” (RPRO), con 43, 38 y 30 unidades de significado respectivamente. En la Tabla 1 se presenta la distribución de frecuencias de las unidades de significado de cada una de las categorías codificadas y analizadas, en función de los factores que intervienen en la integración y las competencias específicas de la profesión que se logran con los contenidos de los Módulos Bases Morfológicas I y II.

Nivel 2: Se agruparon y estructuraron las 3 categorías que emergieron en el primer nivel; posterior a un proceso de comparación intercategorías en el que se buscaron similitudes estructurales y elementos comunes de este análisis, emergieron 2 núcleos temáticos o metacategorías en torno a los cuales se reagruparon todas las categorías (Tabla 2).

Nivel 3: Posterior al análisis secuencial y transversal de las metacategorías, surge el dominio cualitativo "Consideraciones para favorecer la integración entre Bases Morfológicas I y II con la Línea Profesional en el desarrollo de competencias disciplinares para el futuro desempeño laboral".

\section{DISCUSIÓN Y CONCLUSIÓN}

De acuerdo a los objetivos del estudio al pre- tender develar los factores que intervienen en la integración entre Bases Morfológicas I y II con la Línea Profesional, se observa que los informantes identifican sólo aquellos que la dificultan, destacando la no participación en la planificación educativa de la asignatura y la actitud de los involucrados en el proceso (estudiantes, profesores de Ciencias Básicas y Enfermeras). Ello podría ser el resultado que los profesores encargados son académicos expertos en esas materias, desconocedores de las competencias específicas de la profesión (8) y probablemente una invalidación por parte de estos académicos hacia las enfermeras, avalado por los discursos de los informante claves. Otra causa está relacionada a los docentes de Ciencias Básicas, quienes se resisten al nuevo paradigma educacional implementado por la Carrera $(11,12)$, reflejado en la actualidad en que los contenidos se imparten en forma separada, es decir tres asignaturas en una: Anatomía, Histología y Embriología.

Si bien es cierto que los informantes claves no identifican factores que contribuyan a la integración entre ambas líneas curriculares, sí emergen en sus discursos propuestas para el mejoramiento de éstas, las que se orientan a la participación en la "planificación educativa”, desde la organización de la actividad hasta el cumplimiento del paradigma educacional. De ahí la importancia de la planificación educativa integrada (2) para lograr un aprendizaje significativo (1). Es preocupante que las enfermeras no identifiquen aspectos favorecedores, ya que el modelo educativo está instaurado desde el año 2003 en todo el Plan de Estudio. Este último factor se podría atribuir a la sensación continua de las enfermeras de ser descalificadas por los académicos de Ciencias Básicas, cuando tenían reuniones de trabajo para la integración de Ciencias Básicas con la línea profesional.

En cuanto a las competencias específicas de la profesión que se logran con los contenidos de los Módulos Bases Morfológicas I y II, Anatomía e Histología las identifican como 


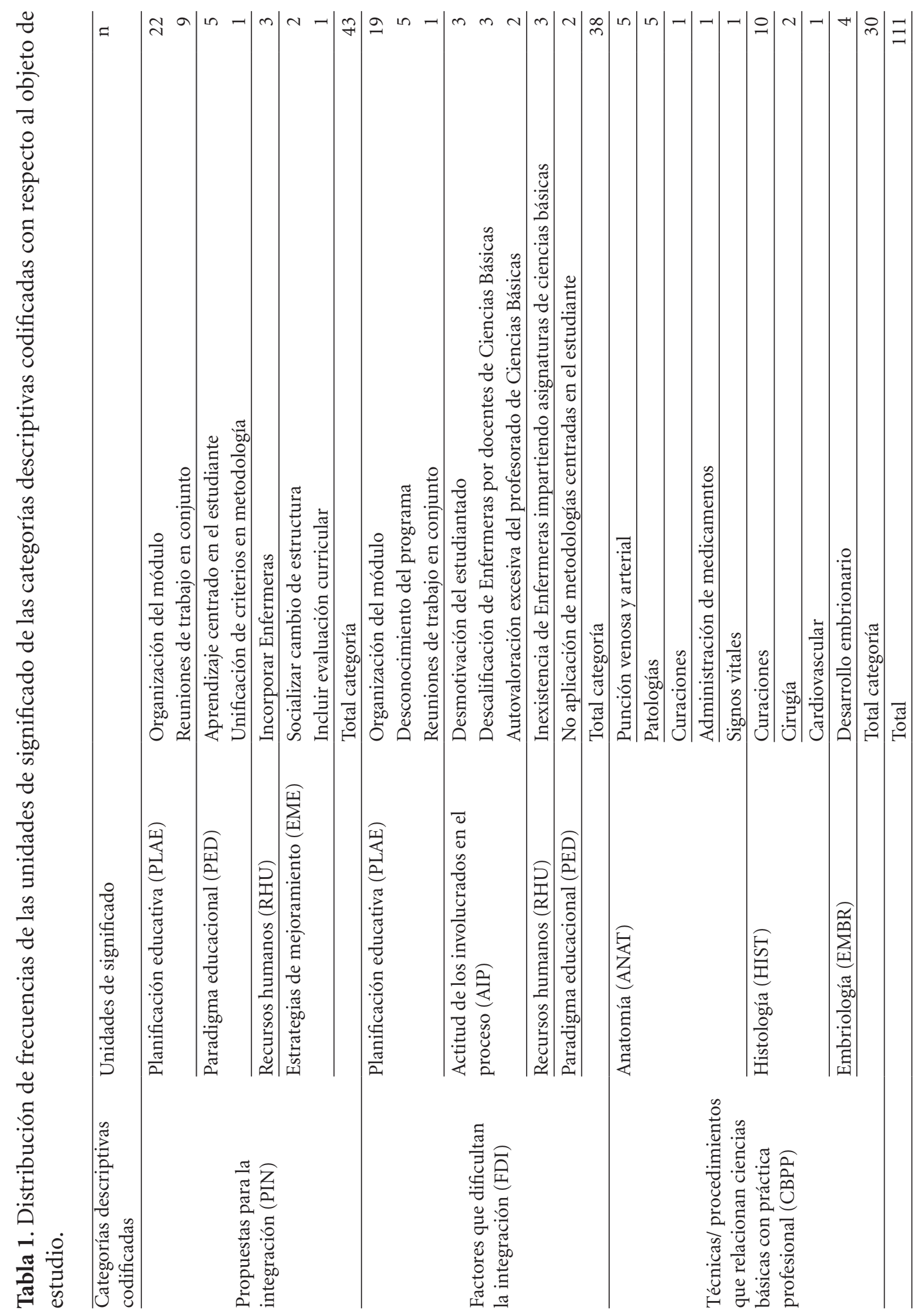


Tabla 2. Distribución porcentual entre metacategorías, categorías descriptivas codificadas y unidades de significado con respecto al objeto de estudio.

\begin{tabular}{llcc}
\hline Metacategorías & Categorías descriptivas codificadas & \multicolumn{2}{c}{$\begin{array}{c}\text { Unidades de } \\
\text { significado }\end{array}$} \\
\cline { 2 - 4 } & & $\mathrm{n}$ & $\%$ \\
\hline $\begin{array}{l}\text { Requerimientos de la integración de } \\
\begin{array}{l}\text { contenidos de Morfofunción con la línea } \\
\text { profesional }\end{array}\end{array}$ & \begin{tabular}{l} 
Propuestas para la integración (PIN) \\
\cline { 2 - 4 } $\begin{array}{l}\text { Competencias de la profesión adquiridas } \\
\text { con los contenidos de Morfofunción }\end{array}$
\end{tabular} & $\begin{array}{l}\text { Técnicas/procedimientos que relacionan } \\
\text { ciencias básicas con práctica profesional (CBPP) }\end{array}$ & 38,7 \\
\hline & & 38 & 27 \\
\hline
\end{tabular}

fundamentales para la realización de algunas técnicas y procedimientos propios de la actividad profesional. Lo develado confirma la importancia de una base de conocimientos para la aplicación del Proceso Enfermero en todas sus etapas (7), al igual que la motivación de los estudiantes al relacionar en forma inmediata lo aprendido con su futuro quehacer profesional (6). Llama la atención que los contenidos de Embriología no son considerados como base para el desarrollo de la línea profesional, lo que puede deberse a que son temas que se integran en el Módulo de Enfermería Materno-Infantil, y que lo imparten enfermeras en el cuarto año de la Carrera, lo que reafirma la relevancia de materializar el conocimiento adquirido lo más próximamente posible y evidencia la desvinculación entre contenidos de Morfología y los de otras asignaturas (5).

Con todo lo explicitado anteriormente, se concluye que no se ha logrado la integración ya que los contenidos son impartidos en forma separada, más aún en el primer año, tampoco existe unión con la línea profesional, confirmándose la observación empírica que dio origen a esta investigación.

Queda el desafío de consultar al principal participante del proceso enseñanza-aprendizaje que son los estudiantes. También surge una nueva meta de elaborar una propuesta para delimitar los contenidos en pos de contribuir a la eficacia en la formación.

\section{REFERENCIAS}

1. Ausebel D, Novak J, Hanesian H. Psicología Educativa. Un punto de vista cognoscitivo. $2^{\text {da }}$ ed. México, D.F.: Trillas; 2000. p. 47.

2. Cañizares O, Sarasa N, Labrada C. Enseñanza integrada de las Ciencias Básicas Biomédicas en Medicina Integral Comunitaria. Educ Med Super [Internet]. 2006 Mar [citado 3 marzo 2015]; 20(1): Disponible en: http://scielo.sld.cu/scielo.php? script $=$ sci_arttext $\&$ pid $=$ S0864 21412006000100005\&lng=es

3. Rosell W, Dovale C, Álvarez I. Características del libro de texto básico cubano de Morfología Humana. Educ Med Super [Internet]. 2004 Jun [citado 12 marzo 2015 ] ; 18(2): 1-1. Disponible en: http://scielo.sld.cu/scielo. php?script $=$ sci_arttext $\&$ pid $=$ S0864 21412004000200003\&lng=es

4. Díaz-Velis E, Ramos R, Mendoza C. Un reclamo necesario, la integración de los contenidos en la carrera de Medicina. Educ Med Super [Internet]. 2005 Mar [citado 10 mar 2015]; 19(1): 1-1. Disponible en: http://scielo.sld.cu/scielo. php? script $=$ sci_arttext $\&$ pid $=$ S0864 21412005000100002\&lng=es

5. Rosell W. Aspectos fundamentales de la vinculación básico-clínica en los planes 
de estudios de Medicina. Educ Med Super. 1987; (1-2): 31-7.

6. Rosell W, Dovale C, González B. La enseñanza de las Ciencias Morfológicas mediante la integración interdisciplinaria. Educ Med Super [Internet]. 2004 Mar [citado 11 marzo 2015]; 18(1): Disponible en: http://scielo.sld.cu/scielo.php?script $=$ sci_arttext\&pid $=$ S0864 21412004000100003\&lng=es

7. Dionisio de Cavalier M, Chalub D. El aprendizaje significativo de las ciencias morfológicas en medicina: experiencia y aportes para su enseñanza en clínica dermatológica. Int. J. Morphol. 2009; 27(2): 565-9.

8. Universidad de La Frontera-UFRO. Modificación Plan de estudios y reglamento Carrera de Enfermería: resol ex 2044 del 30 dic 2003.Temuco: UFRO; 2003.

9. Carretero M. Constructivismo y educación. Buenos Aires: Paidós; 2009. 222 p.

10. Fullan M. The meaning of educational change. New York: Teacher College Press; 1991. p. 5-6.

11. Fullan M. Research into Educational Innovation. En Glatter R, Preedy M, Rickes C, eds. Understanding School Management. Milton Keynes, U.K.: Open Univ. Press; 1987. p. 195-211.

12. Universidad de La Frontera-Carrera de Enfermería. Programa Bases Morfológicas I MBA 107. Temuco: UFRO; 2011.

13. Universidad de La Frontera-Carrera de Enfermería. Programa Bases Morfológicas II MBA 125. Temuco: UFRO; 2011.

14. Universidad de La Frontera. Evaluación de la docencia. Intranet. Temuco: UFRO; 2011.

15. Universidad de La Frontera. Seguimiento de Asignaturas y estudiantes. Programa Asignatura. Temuco: UFRO; 2011.

16. Segura N, Gómez EI, Machado ML, Cruz J, Valdés C, Enríquez H.. Vinculación interdisciplinaria, semántica y de contenidos, durante el primer semestre de la licenciatura en enfermería. Educ Med
Super. 2001; 15(3): 258-267.

17. Arenas NM. Una metodología alternativa para la enseñanza de la anatomía en los estudios de enfermería de la Universidad de Carabobo. Rev Lat Am. Enfermagem. 1993; 1(1): 73-83.

18. Escalona D, Leyva J. Importancia del conocimiento de la anatomía y su contribución en la aplicación del Proceso de Atención de Enfermería. Alfinal.com [monografía en Internet]. 2006 [citado 31 enero 2013]. Disponible en: http://www.alfinal. com/Cuba/enfermeria.php

19. Schencke C. Hidalgo A. Comparación de dos Softwares en Histología Humana, como Utilización Complementaria a la Enseñanza Tradicional. Int. J. Morphol. 2011; 29(4): 1388-1393.

20. Falcó A. Orientar la asignatura de Anatomía y Fisiología hacia las competencias profesionales de enfermería. Propuesta de un proyecto docente. Educ. méd. 2005; 8(4): 208-215.

21. Guevara MC, González N, Cañizares O, Carbó J. Perfeccionamiento del proceso docente educativo: una experiencia en la disciplina Ciencias Morfológicas. Archivo Médico de Camagüey [Internet]. 2004 febr [citado 31 enero 2013]; 4(3): [aprox. 4 p]. Disponible en http://www.amc.sld. $\mathrm{cu} / \mathrm{amc} / 2000 / \mathrm{v} 4 \mathrm{n} 3 / 356 . \mathrm{htm}$

22. Carbonell SA, Barrios L, Vásquez O. Bases teóricas para la integración de las ciencias básicas biomédicas en una disciplina: Theoretical bases. Educ Med Super [Internet]. 2010 Sep [citado 10 oct 2014]; 24(3): 344-351. Disponible en: http://scielo.sld.cu/scielo. php?script $=$ sci_arttext\&pid $=$ S0864 21412010000300006\&lng=es

23. Kyriacou C. Essential teaching skills. Londres: Nelson Thornes; 2007; p. 44.

24. Stake R. Investigación con estudio de casos. $2^{a}$ ed. Madrid: Morata. 1999; p. 94103.

25. Latorre A. La investigación-acción: conocer y cambiar la práctica educativa. Bar- 
celona: Graó. 2003; p. 179.

26. Polit D, Hungler B. Investigación Científica en Ciencias de la Salud. 6a ed. México: Mc Graw-Hill Interamericana; 2000. p. 725.

27. Glaser B, Strauss A. El método de comparación constante de análisis cualitativo. In The discovery of Grounded Theory: strategies for qualitative research. New York: Aldine; 1967. p. 101-115.

28. Gil J. Análisis informatizado de datos cualitativos. Sevilla: Kronos; 2001.p. 152.

29. Miles MB, Huberman A. Qualitative data analysis: an expanded sourcebook. Newbury Park, CA: Sage. 1994; p. 352.

30. Latorre A, Del Rincón D, Arnal J. Bases metodológicas de la investigación educativa. Barcelona: Hurtado. 1996; p. 49-50.
31. Guba EG, Lincoln YS. Effective evaluation: improving the usefulness of evaluation results through responsive and naturalistic approaches. San Francisco: Jossey-Bass. 1981; 423 p.

32. Lolas F, Quezada A, eds. Pautas Éticas de Investigación en Sujetos Humanos: Nuevas Perspectivas. Santiago, Chile: Programa Regional de Bioética OPS/OMS; 2003.

33. González M. Aspectos éticos de la investigación cualitativa. Revista Iberoamericana de Educación. 2002 [citado 10 noviembre 2010]; (29): 85-103. Disponible en: http://www.umch.edu.pe/acreditacion/download/08\%20Aspectos $\% 20$ eticos $\% 20$ de $\% 201$ a $\% 20$ investigacion $\% 20$ cualitativa.PDF 